\title{
Le séquençage de nouvelle génération (Next-Generation Sequencing, ou NGS) appliqué au diagnostic de maladies monogéniques hétérogènes
}

\section{Notions essentielles pour le dialogue entre cliniciens et généticiens}

\author{
Martin Krahn, Nicolas Lévy, Marc Bartoli
}

Le dialogue entre généticiens et cliniciens est devenu plus que jamais nécessaire avec l'implémentation du NGS pour le diagnostic génétique de pathologies hétérogènes. Une interaction étroite permet d'optimiser les possibilités d'un diagnostic précis, en retenant l'implication d'un gène dans la pathologie que présente le patient, par l'interprétation des mutations souvent nombreuses mises en évidence par les analyses de NGS dans une diversité importante de gènes. Pour cela, une connaissance synthétique du processus de NGS est dorénavant nécessaire en pratique clinique. Ce processus comporte de multiples étapes de génération et d'analyse des données, associées à un vocabulaire spécifique, dont nous souhaitons présenter les notions essentielles dans cette Fiche Pratique.

La commercialisation depuis 2005 des technologies de NGS a révolutionné au cours de ces dernières années la dimension des analyses génétiques par un changement majeur d'échelle des capacités de séquençage.

Ayant trouvé rapidement de nombreuses applications dans le domaine de la recherche, en particulier pour l'identification de nouveaux gènes impliqués dans des maladies monogéniques, le NGS a progressivement été validé pour des applications en diagnostic génétique.

Le NGS repose sur la génération massive de données de séquences obtenues par des cycles successifs d'incorporation de nucléotides, et ainsi l'émission de signaux qui sont ensuite convertis en information de séquence. Différentes technologies existent actuellement, notamment basées sur un séquençage en parallèle de millions de molécules d'ADN, avec une augmentation toujours croissante des capacités de séquençage associée à une diminution progressive des coûts, et de nouvelles approches sont en développement (en particulier le séquençage direct de molécules d'ADN uniques).

De manière schématique, le processus de NGS est constitue de multiples étapes de génération et d'analyse des données, avec la prise en compte de critères de qualité de séquençage (en particulier l'analyse de la "couverture" et de la "profondeur de lecture" de la séquence d'intérêt), qui sont présentées de manière synthétique dans la figure en association avec des termes d'usage courant associés.

Le NGS permet dorénavant d'effectuer l'analyse de régions d'intérêt de grande taille, ce qui n'était pas possible avec le séquençage "classique" (méthode de Sanger) utilisé depuis les années 1980, en raison de limitations de coûts et de débit de quantités de séquences pouvant être générées, ce qui restreignait son application à des approches de séquençage "gène après gène ", responsable dans de nombreux cas d'une longue errance diagnostique.

Avec une multiplication des capacités de séquençage dans un rapport de plusieurs dizaines voire centaines de milliers de fois par rapport au séquençage Sanger, le NGS a permis le développement de nouvelles stratégies d'analyses mutationnelles, dont trois principales sont actuellement utilisées :

- Analyse de "listes de gènes" ou "panel de gènes": il s'agit de l'analyse simultanée de la séquence d'un certain nombre de gènes d'intérêt (habituellement une ou plusieurs dizaines). Comme pour le séquençage Sanger, l'analyse est habituellement centrée sur les régions codantes des gènes et les bornes introniques flanquantes, où est localisée 


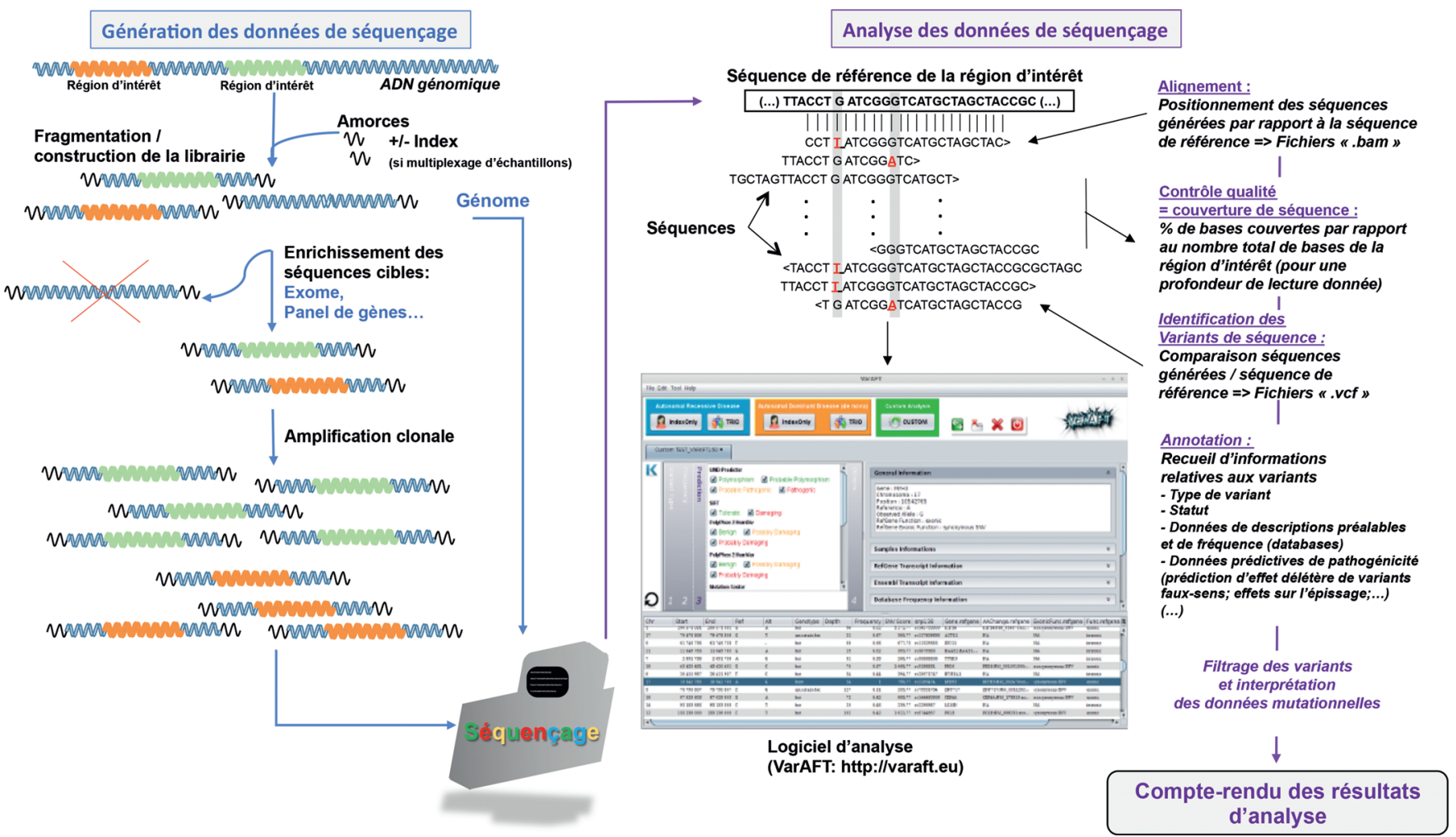

Figure 1

Principales étapes de génération et d'analyse de données de NGS. Les principales étapes de génération et d'analyse de données de NGS sont schématisées dans le cadre des techniques actuelles de séquençage parallèle à haut débit, avec une définition des principaux termes d'usage courant associés.

la majorité des mutations délétères. L'approche "panel de gènes " est actuellement la plus utilisée en diagnostic génétique, notamment pour analyser des listes de gènes connus comme étant impliqués dans un groupe de pathologies (par exemples les dystrophies musculaires, ou encore les myopathies ou les neuropathies périphériques au sens large). Certains "panels de gènes" très larges sont commercialisés et comportent la majorité des gènes répertoriés comme étant impliqués en pathologie humaine (plus de 4 000), dans la base de données OMIM (Online Mendelian Inheritance in Man, www.omim.org). Ces "super-panels" sont également appelés "exomes cliniques".

- Analyse "d'exome" ("Whole Exome Analysis/ WES ") : cette approche consiste en l'analyse simultanée de la totalité des séquences codantes (et bornes introniques flanquantes) de tous les gènes du génome (environ 20 000), correspondant à environ $1 \%$ du génome, soit environ 180000 exons et 30 millions de paires de bases.

- Analyse du génome dans son intégralité ("Whole Genome Analysis "/WGS) soit 3 milliards de bases, comprenant les séquences codantes et non codantes.
Il est à noter que certains laboratoires ont choisi de mettre en place des stratégies de séquençage "large" (panel OMIM, exome, voire génome pour certains laboratoires pionniers anglo-saxons), puis d'effectuer un filtrage restreint sur liste de gènes d'intérêt, ce qui permet une "ouverture" du filtre d'analyse informatique à une liste plus grande en cas de résultat initial non concluant. Le choix de la stratégie prend actuellement en compte notamment les capacités des séquenceurs dont disposent les laboratoires, et en lien avec ceci les coûts de l'analyse.

La conséquence directe de l'augmentation des capacités de séquençage a été l'augmentation considérable des données mutationnelles à interpréter. À titre d'exemple, l'analyse de l'exome d'un individu génère en moyenne 25000 variants par rapport à la séquence de référence du génome humain. L'objectif étant d'identifier la ou les mutation(s) délétères responsable(s) de la pathologie que présente le patient, le défi consiste à recueillir un maximum d'informations disponibles pour chacun des variants de séquence identifiés, et effectuer une interprétation à deux niveaux : juger du caractère pathogène ou non des variants, puis de leur lien avec la 
pathologie concernée. Cette étape essentielle de l'analyse, appelée "annotation ", permet grâce à des logiciels spécifiques de compiler des informations très diverses (type de variant, statut hétérozygote/homozygote/hémizygote, données de description et de fréquence de bases de données mutationnelles, données bioinformatiques prédictives de pathogénicité, données de ségrégation familiale, etc.), qui permettront d'appliquer des filtres de tri. Malgré l'utilisation de filtres, la conclusion n'est pas toujours évidente, et la validation de l'implication d'une/de mutation(s) d'un gène dans la pathologie que présente le patient est largement optimisée par une discussion conjointe des résultats entre généticiens et cliniciens. Néanmoins, le taux de diagnostic positif obtenu pour des analyses en NGS de cas index sporadiques reste limité à 25-50\%, selon les pathologies, avec des résultats équivalents pour les approches "panel" ou "exome". Selon des études récentes, une relative amélioration est obtenue par le séquençage de génome dans sa totalité, sachant qu'une proportion probablement importante d'événements mutationnels délétères reste pour l'instant "difficilement accessible" en raison d'éléments insuffisants pour l'interprétation, en particulier pour des variants en régions noncodantes.

Avec le changement d'échelle associé aux analyses NGS, les comptes rendus d'analyse, et l'information à apporter aux patients se sont également complexifiés. À l'initiative de la Fondation Maladies Rares, un groupe d'experts a élaboré une notice d'information et un modèle de consentement à vocation nationale (disponibles en téléchargement : http://fondationmaladiesrares.org/actualite/consentement-a-letude -des-caracteristiques-genetiques), prenant en compte le contexte du NGS. Ces documents abordent en particulier la problématique des identifications " fortuites/ accidentelles " (variants "supplémentaires " dans des gènes analysés en lien avec la pathologie que présente le patient, appelées identifications "non-sollicitées " ; ou sans lien, appelées " identifications secondaires").
Afin de clarifier l'offre diagnostique par NGS, des réflexions ont été engagées dans le cadre des différentes filières nationales de maladies rares, et notamment FILNEMUS. L'objectif est de proposer sur le plan national une démarche homogène consistant en l'analyse de listes de gènes par groupe de pathologies, établies de manière conjointe entre généticiens et cliniciens, et utilisées de manière consensuelle par les laboratoires diagnostiques. Dans le cadre d'une démarche séquentielle, l'analyse peut notamment être réalisée dans un premier temps sur une liste restreinte de gènes, puis élargie en cas de résultat négatif ou non concluant à une liste plus importante, et finalement une analyse de l'exome (voire génome). De la même manière, un enjeu majeur réside en l'homogénéisation des démarches d'interprétation du caractère pathogène ou non des variants identifiés.

Le NGS est maintenant implanté dans les laboratoires diagnostiques. L'instauration récente du Référentiel des actes Innovants Hors Nomenclature (RIHN) déterminant la tarification des analyses de NGS, et la réflexion actuelle sur la mise en place de plateformes nationales de séquençage à très haut débit permettront de systématiser à l'avenir l'accès à ces technologies pour la prise en charge des patients.

Next-generation sequencing (NGS) applied to diagnosis of heterogeneous genetic disorders: essential concepts for dialogue between clinicians and geneticists

\section{LIENS D'INTÉRÊTT}

Les auteurs déclarent n'avoir aucun lien d'intérêt concernant les données publiées dans cet article.

\section{RÉFÉRENCES}

1. Krahn M, Arveiler B. Le séquençage de nouvelle génération : principe, applications en diagnostic et perspectives. In : Livre national d'enseignement - Génétique Médicale - DFGSM2/3. Issy-les-Moulineaux : Elsevier-Masson, 2016.

2. Gorokhova S, Biancalana V, Lévy N, Laporte J, Bartoli M, Krahn M. Clinical massively parallel sequencing for the diagnosis of myopathies. Revue Neurologique (Paris) 2015 ; 171 : 558-71. 